FedUni ResearchOnline

https://researchonline.federation.edu.au

This is the peer-reviewed version of the following article:

Kahandawa, G., Epaarachchi, J., Wang, H., Canning, J., \& Lau, A. (2013).

Extraction and processing of real time strain of embedded FBG sensors using a fixed filter FBG circuit and an artificial neural network. Journal of the International Measurement Confederation, 46(10), 4045-4051.

Which has been published in final form at: https://doi.org/10.1016/j.measurement.2013.07.029

Copyright @ 2014 Elsevier B.V. All rights reserved. 


\title{
Extraction and processing of real time strain of embedded FBG sensors using a fixed filter FBG circuit and an artificial neural network
}

\author{
Gayan C. Kahandawa a,*, Jayantha Epaarachchi ${ }_{\perp}^{a}$, Hao Wang a, John Canning ${ }_{\perp}^{b}, K_{\Lambda}$ K.T. Lau ${ }_{\perp}^{c}$ \\ 2 a Centre of Excellence in Engineered Fibre Composites, University of Southern Queensland, Australia \\ ${ }^{\mathrm{b}}$ Interdisciplinary Photonic Laboratories, School of Chemistry, University of Sydney, Australia \\ ${ }^{\mathrm{c}}$ The Hong Kong Polytechnic University, Hong Kong
}

\section{A R T I C L E I N F O}

\section{Article history:}

Received 12 November 2012

Received in revised form 18 July 2013

Accepted 23 July 2013

Available online $\mathrm{xxxx}$

\section{Keywords:}

FBG sensors

Composite structures

Structural health monitoring

\begin{abstract}
A B S T R A C T
Fibre Bragg Grating (FBG) sensors have been used in the development of structural health monitoring (SHM) and damage detection systems for advanced composite structures over several decades. Unfortunately, to date only a handful of appropriate configurations and algorithm sare available for using in SHM systems have been developed. This paper reveals a novel configuration of FBG sensors to acquire strain reading and an integrated statistical approach to analyse data in real time. The proposed configuration has proven its capability to overcome practical constraints and the engineering challenges associated with FBG-based SHM systems. A fixed filter decoding system and an integrated artificial neural network algorithm for extracting strain from embedded FBG sensor were proposed and experimentally proved. Furthermore, the laboratory level experimental data was used to verify the accuracy of the system and it was found that the error levels were less than $0.3 \%$ in predictions. The developed SMH system using this technology has been submitted to US patent office and will be available for use of aerospace applications in due course.
\end{abstract}

(c) 2013 Published by Elsevier Ltd.

\section{Introduction}

The aerospace, space and many other high-tech industries have increasingly replaced metallic parts with components made from various types of fibre reinforced composites (FRC). As such, FRC parts are frequently found in recently developed primary load-bearing structures in many applications such as structural components of the Boeing 787 and Airbus 380. However, there are many critical issues such as difficulty of repair, anisotropic behaviour, apparent degradation of properties with time, expensive infrastructure, and the complex nature of failure and damage accumulation and failure modes which are not completely resolved to date. Due to the critical and unresolved nature of damage modes, the FRC components must

\footnotetext{
* Corresponding author. Tel.: +61 466267946; fax: +61 746312110.

E-mail address: gayan@usq.edu.au (G.C. Kahandawa).
}

be closely monitored to prevent unexpected catastrophic failure.

In structural applications there are many notorious stress-concentrated regions such as pin-loaded holes, joints, connections and critical geometrical shapes which are pronto induce damage that concurrently include splits, transverse cracks and delamination [1-3]. These damages are hardly visible and monitoring the stress levels, as much as possible at the critical areas is essentiâ to guarantee structural integrity and comply with highly restrictive codes and standards. As a consequence, damage diagnostic and damage prognostic SHM systems are being developed for such composite structures [4,5].

To identify and address complex failure criteria inherent in FRC materials, the SHM systems being developed for composite structures must be robust [6].The damage accumulation in each layer of a composite laminate is primarily dependent on the properties of the particular layer 
$[7,8]$, and the loads which are imparted onto the layer. The anisotropic property of laminated composite is one of the major reasons to have embedded sensors measuring structural behaviour of laminate composite materials. A surface mounted sensor network is not sufficient for health monitoring of composite structures. The Fibre Bragg Grating (FBG) sensor is one of the most suitable sensors for laminated composite structures as the FBG sensors can be embedded in FRP composites during the manufacture of the composite part with no effect on the strength of the part as the size of the sensor is diminutive. This sensor is suitable for networking since it has a narrowband response with a wide wavelength operating range, hence can be highly multiplexed. As a nonconductive sensor it can also operate in electromagnetically noisy environments without any interference.

The use of embedded FBG sensors has been a major consideration for SHM systems and damage identification in composites since their invention in 1987 [9-13]. Generally, the FBG spectrum will be distorted due to uneven strain distribution over its gauge length. Close observation of the distorted spectrum provides a significant amount of detail about the strain distribution around the embedded sensor location. Observations of the distorted spectrums produced by these sensors have been used to estimate the damage in composites. The majority of research works have focused on the investigation of the spectra of embedded FBG sensors under simple unidirectional loading.

However, in reality, the applied loads are not limited to uni-axial loads, and hence, the performance of FBGs in multi-axial loading situation has to be investigated for comprehensive damage characterization. The FBG spectral response is significantly complicated by multi-axial loading conditions [14], fibre orientation, and the type of damage present in the structure [15]. As a result, the cause of the distortion of FBG spectra not only depends on the consequences of accumulated damage but also loading types and the fibre orientation. For example, Kahandawa et al. has shown that the application of tensional loads caused substantial distortions to the spectra of FBGs which were embedded between non-parallel fibre layers. These issues create a serious need to identify and exclude those discrepancies from any SHM system for composite structures.

This paper details exclusive research work undertaken to eliminate the non-relevant data contained in embedded FBG spectra and process relevant damage data for SHM systems for composite structures. The proposed procedure eliminates the need for expensive and bulky equipment used in traditional data acquisition systems, and significantly shortens data processing time. The system introduced here can be used to capture the distortions of reflected spectra of an embedded FBG sensor in a composite laminate, and quantitatively estimate the distortion to the spectra.

\section{Fixed filter decoding system for FBG sensors}

In recent years many systems for decoding FBG spectrums using fixed FBG filters have been developed [1620]. Fig. 1 illustrates a general arrangement of a fixed
FBG filter system. This system consists of a tunable laser (TLS), fixed FBG filter, optical couplers (CP) and photo detector (PD). A high frequency data acquisition system (DAQ) was used to record the PD voltage values.

The system shown in Fig. 1 is a building block of the proposed decoding system, which uses only one FBG filter. A tunable laser light is transmitted into the FBG sensor and the reflected spectrum of the FBG sensor is fed to the FBG filter through a coupler of a ratio 50:50. The intersection of the wavelengths reflected from the sensor and the filter is reflected to the photo-detector. The sensor receives the total wavelength range from the tunable laser source, while the filter receives only the wavelengths reflected by the sensor. Therefore, the filter can only reflect light to the photo-detector if the wavelength from the sensor is lying within the filter's grating range $\left(\lambda_{1}\right)$. The reflected light of the filter is captured using the photo-detector, converted to a voltage, and recorded in the DAQ system in time domain. This building block can be used to build a large system of many wavelengths as required by the application.

Fig. 2 shows the reflected spectra of the FBG sensor and the filter. The filter can only reflect light if the input light wavelength from the sensor lies within the filter's wavelength range. Thus, the filter reflects the intersection as shown in Fig. 2.

The reflected light of the FBG filter was captured using the PD, and the PD's output voltage was recorded using the DAQ in time domain. Fig. 3 shows the PD voltage in the time domain corresponding to the intersection of the spectrums shown in Fig. 2. In addition, the reflected spectra of the FBG also can be viewed in cathode ray oscilloscope or recorded as voltage in time domain. By matching the tunable laser swept frequency with the DAQ sampling frequency, it is possible to estimate the spectra in wavelength domain.

Since the filter is fixed, the intersection of two spectra depends on the fixed sensor's position. Variation of the intersection is proportional to the shift of wavelength of grating due to applied strain and/or the distortion. If more filters are used, the bandwidth of filters can be increased and the readable range of the sensor wavelength will be increased.

There were several attempts to fit the FBG spectra using mathematical functions such as the commonly used Gaussian curve fit [21]. Unfortunately, Gaussian fit always gives an error for a distorted spectrum. Recently Kahandawa et al. has shown that a distorted spectrum can be considered as a piece wise continuous function, $f_{p c}$ and the area under each piece of the spectrum $P$ can be obtained using the following integral [22]:

$P=\beta \int_{t_{1}}^{t_{2}} f_{p c} d t$

where $\beta$ is a constant dependent on the power of the source, and $t_{1}$ and $t_{2}$ are the integral limits in the time domain (Fig. 4). Subsequently $P$ can be used for estimation of the strain and the distortion.

The area under the intersection curve which is proportional to the broadening of the spectrum and/or distortion (Fig. 4) can be calculated using numerical integration. 


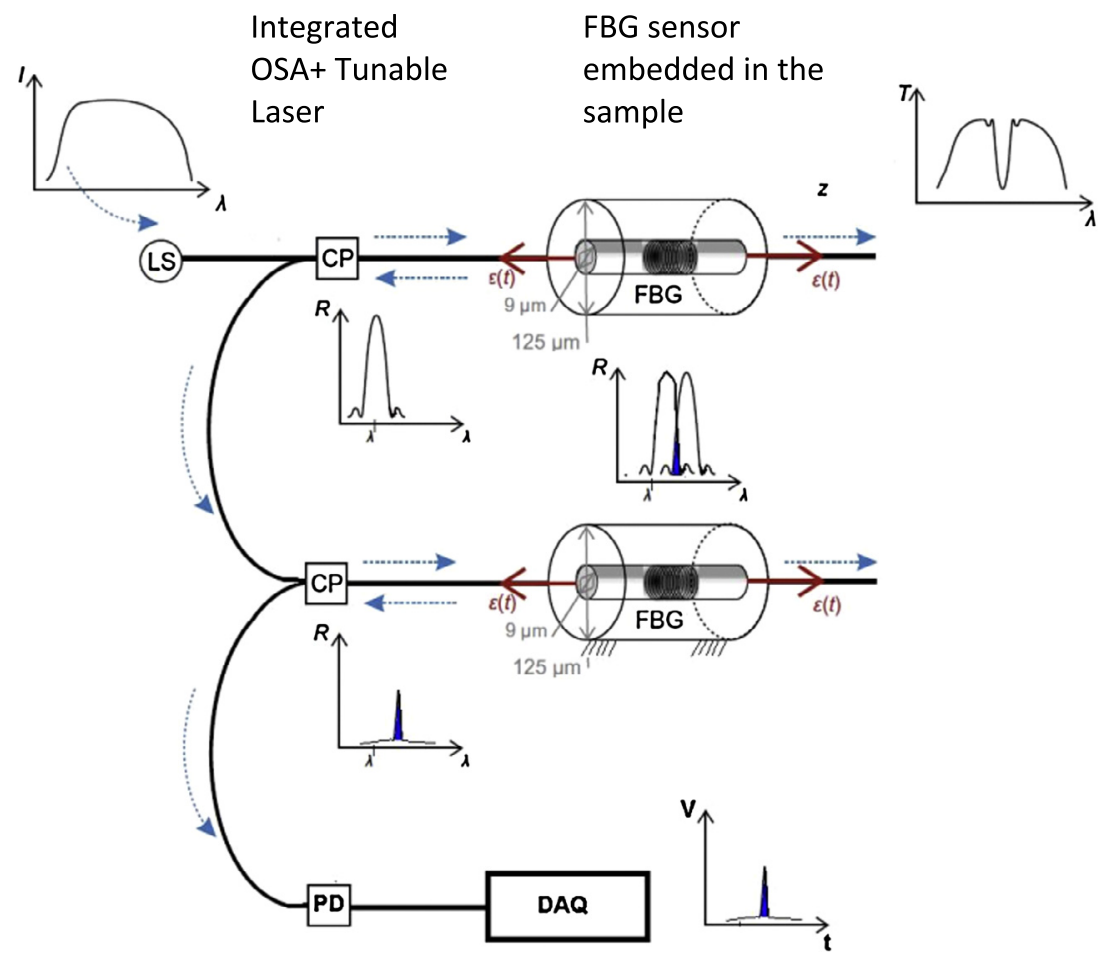

Fig. 1. FBG spectrum decoding system.
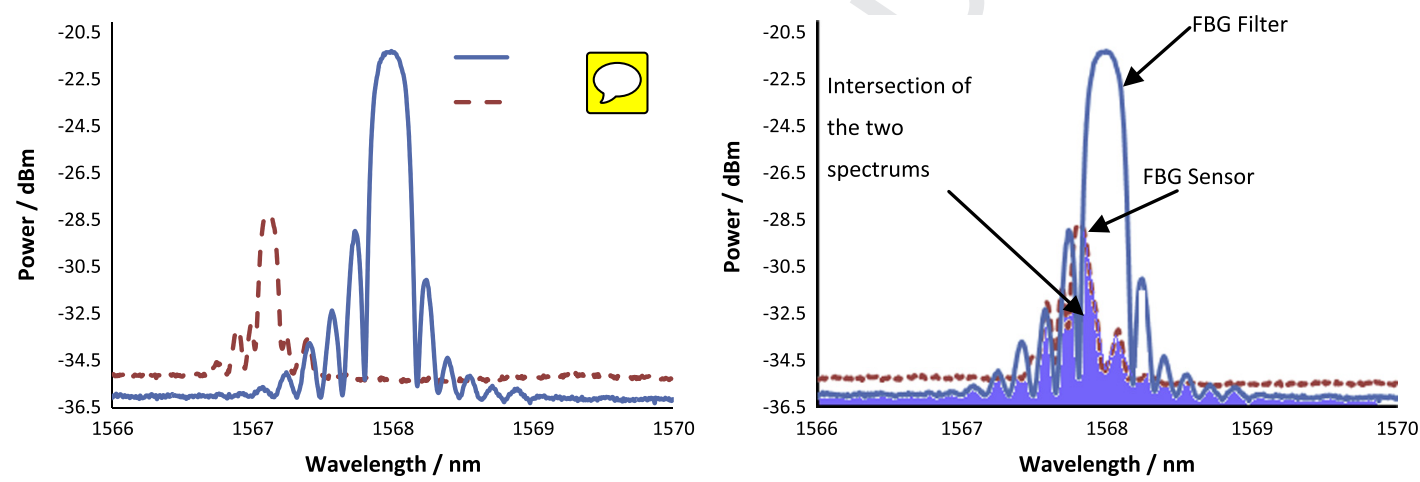

Fig. 2. Intersection of the FBG spectra.

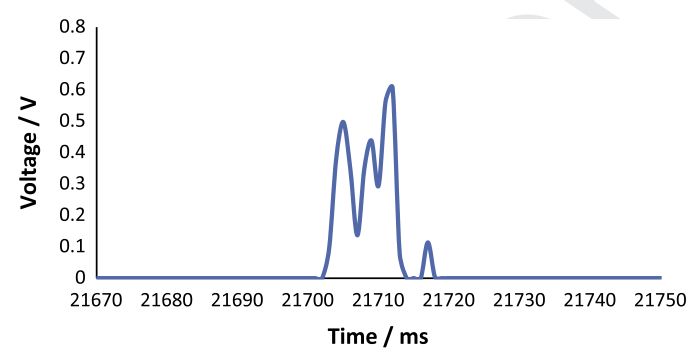

Fig. 3. PD reading due to the intersection of the FBG spectra.
However, the conversion of this calculated area to strain is a significantly difficult mathematical problem and an extensively time consuming process. As a consequence, the conversion of intersection data becomes an extremely difficult task for real time SHM processes. As such a logical mathematically înexpensive procedure was developed to use the calculated area as an input to a decision making intelligent algorithm. The system is able to train itself and predict the strain or alarm when it sees any abnormal input from calculated area. For this case artificial neural network algorithm was selected as the decision making intelligent algorithm.

3. Artificial neural network (ANN) for learning, selftraining and prediction in SḦM systems

Many ANN based algorithms have been developed to use at various stages of SHM systems. Hosni et al. [23] used 
ANN for reliability analysis of structures. They used an ANN of 1 hidden layer and 10 PEs to predict the response of a structure. Mechanical properties, thickness of the plates, angles of orientation and in-plane loads were input to the network. After training, the output of the ANN is

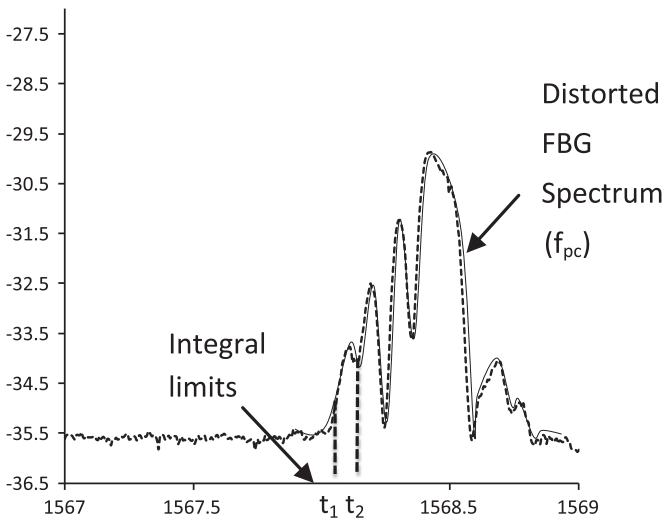

Fig. 4. The piecewise continuous function.

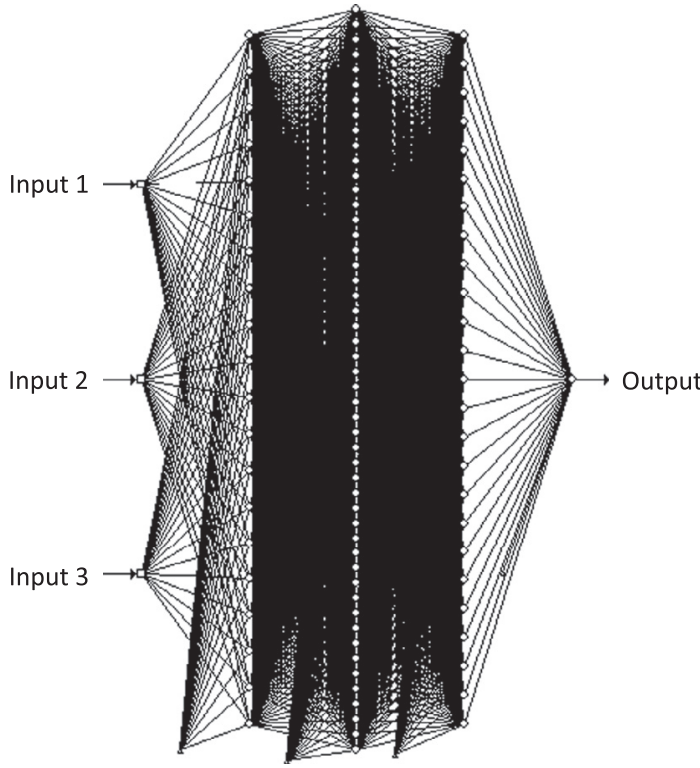

Fig. 5. ANN developed to estimate strain.
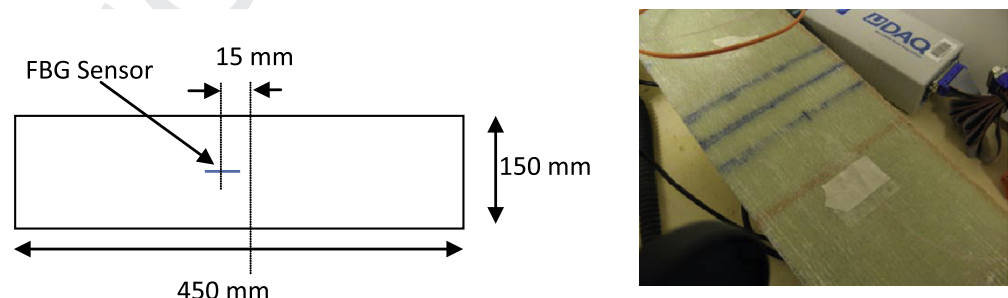

Fig. 6. Sample with embedded FBG sensor. 

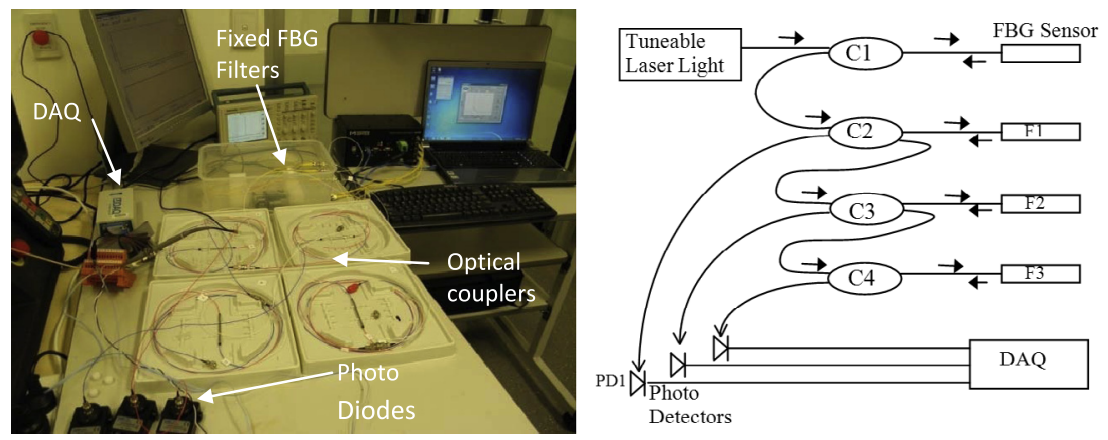

Fig. 7. (a) Optical circuit and the specimen and (b) layout of couplers and PD arrangement.

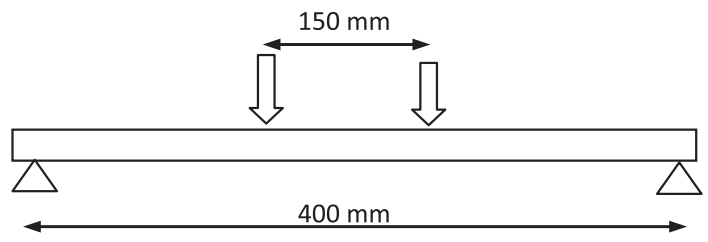

Fig. 8. Experimental setup.

$[0 / 90 / \pm 45 / 90 / 0] s$. Kenetix R246TX epoxy resin was used as the matrix material. An FBG sensor with a wavelength centred $1535 . \hat{2} \mathrm{~nm}$ was embedded between non-parallel layers, 3 and 4, at the location shown in Fig. 6. A specimen was cured at $30{ }^{\circ} \mathrm{C}$ and it was found that the FBG centre wavelength was reduced to $1535.1 \mathrm{~nm}$ due to shrinkage of the sample.

The filter circuit consisted of three filters to cover a wide range of wavelengths (1537.5-1539 nm). A tunable laser light with a swept frequency of $5 \mathrm{~nm}$ per second was connected to the embedded FBG sensor through an optical coupler, $\widehat{\mathrm{C}} 1$. The reflected waveform of the FBG sensor was fed into three FBG fixed filters, F1, F2 and F3 through couplers C2, C3 and C4 respectively. Three PDs were used to read the reflections of the three filters (Fig. 7). The PDs' analogue voltage outputs were recorded using a DAQ at sampling frequency of $10 \mathrm{kHz}$.

The specimen was loaded in a four points bending test rig in steps of $25 \mathrm{~N}$ up to $1000 \mathrm{~N}$ maximum load using an MTS 100 kN uniaxial loadîng machine as shown in Fig. 8.
The readings from the DAQ were recorded at each loading steps.

A detailed finite element model (FEM) was developed for the specimen using the commercial software STRAND7. The model consists with 750 QUAD8 elements. The model was loaded similar to the four point bending experiment. A part of the results of FEA was used to train the ANN and selected data points were kept for experimental validation of the system. Fig. 9 shows the strain contours of layer 4 under flexural loading.

The recorded PD readings were pre-processed using an algorithm written on MATLAB and converted to time domain voltage data. Weighted, pre-processed data was subsequently fed into the ANN through the three input neurons. The ANN was trained using the data set until the RMS error of the network output was reduced to $0.3 \%$.

\section{Results and discussion}

The expected accuracy was reached at 35,000 Epoch. Fig. 10 shows the training rate of the network.

Fig. 11 shows the embedded FBG spectra under 3 loading cases. It can be seen that the distortion has been caused in each spectrum due local and inherent deficiencies [15]. Due to these distortions, estimation of the accurate strain readings is impossible. If there is damage closer to the embedded sensor, the distortion is substantial. However, Fig. 12 shows ANN predictions for various load levels which were not used to train the system. The ANN output is in an excellent agreement with the calculated FEA values.
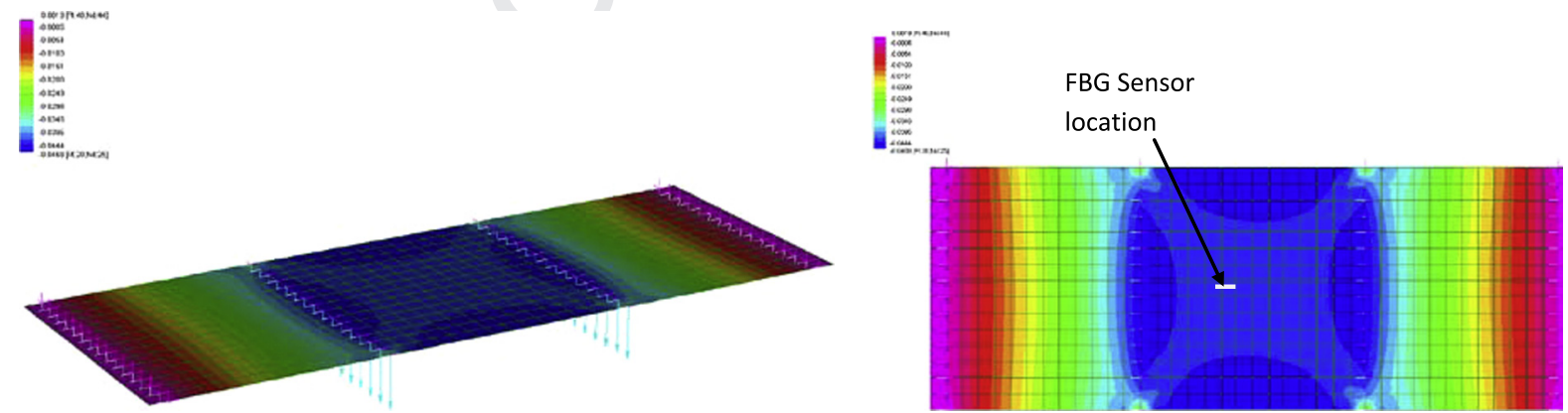

Fig. 9. FEM of the specimen. 


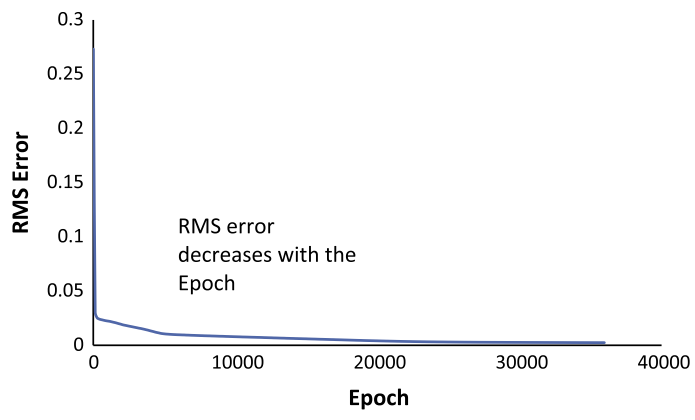

Fig. 10. RMS error with the training process.

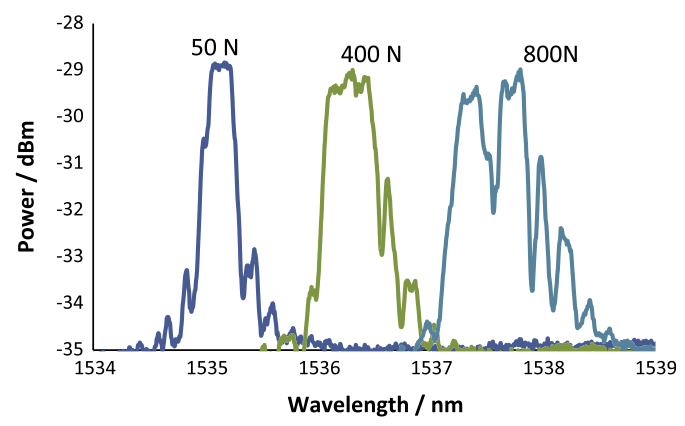

Fig. 11. Distortion to the FBG spectrum with the loading.

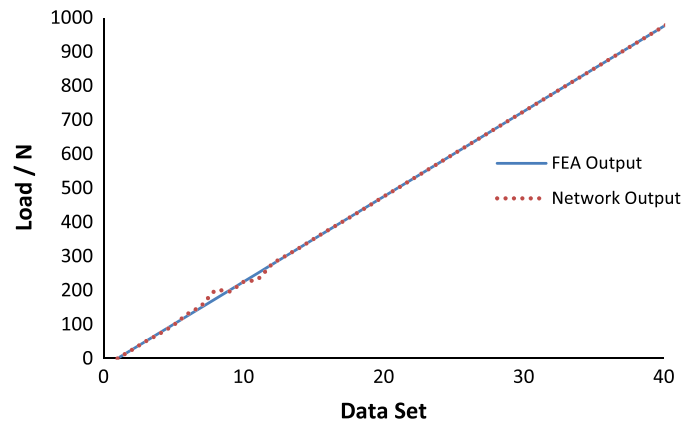

Fig. 12. The network output and the FEA output.

\section{Conclusions}

A spectrum of an embedded FBG sensor, which is distorted due various inherent reasons, has been processed with a fixed filter FBG network. The filtered data was post-processed with an ANN algorithm and strain data was obtained for various load levels. The ANN error level was kept to less than $0.3 \%$. The excellent agreement of ANN predictions and the calculated strains confirmed that the developed ANN system accurately understood the distortions of the spectra induced by the inherent discrepancies of the material. There are some published research work on the use of FBG sensor networks with ANN postprocessing algorithms $[27,28]$. Unfortunately those studies were based on manually read data using specific commercially available equipment which makes it prohibitively difficult to compare the technology detailed in this paper.
The proposed system is a building block for a large network and fundamental to SHM systems. It has been proved that the basic unit has accurately captured the distortion and identified the status of the structure according to its training. The components of this building block, the filters and their outputs are easily integrated into a microprocessor which can condition and post-process through an ANN algorithm to output desired parameter. As such, the integrated system can output real time strain of a composite structure under any operational load level. Unfortunately there are no data from published work available for.

\section{References}

[1] F.K. Chang, K.Y. Chang, A progressive damage model for laminated composites containing stress-concentrations, Journal of Composite Materials 21 (1987) 834-855.

[2] M.T. Kortscho, P.W.R. Beaumont, Damage mechanics of composite materials: I-measurements of damage and strength, Composite Science and Technology 39 (1990) 289-301.

[3] S. Kamiya, H. Sekine, Prediction of the fracture strength of notched continuous fiber-reinforced laminates by interlaminar crack extension analysis, Composites Science and Technology 56 (1996) 11-21.

[4] G. Zhou, L.M. Sim, Damage detection and assessment in fibrereinforced composite structures with embedded fibre optic sensorsreview, Smart Materials and Structures 11 (2002) 925.

[5] F.K. Chang, Structural Health Monitoring, DESTechnol Publications, Lancaster, 2003.

[6] M.S. Reveley, T. Kurtoglu, K.M. Leone, J.L. Briggs, C.A. Withrow, Assessment of the state of the art of integrated vehicle health management technologies as applicable to damage conditions, NASA/TM-2010-216911, 2010

[7] L.N. McCartney, Prediction transverce crack formation in cross-ply laminates, Composites Science and Technology 58 (1998) 10691081.

[8] L.N. McCartney, Prediction of ply crack formation and failure in laminates, Composites Science and Technology 62 (2002) 16191631.

[9] S. Takeda, Y. Okabe, N. Takeda, Application of chirped FBG sensors for detection of local delamination in composite laminates, SPIE, San Diego, CA, United states, 2003. pp. 171-178.

[10] U. Eric, Fibre Optic Smart Structures, John \& Wiley 0050ublications, New York, 1995.

[11] S. Takeda, Y. Okabe, N. Takeda, Delamination detection in CFRP laminates with embedded small-diameter fiber Bragg grating sensors, Composites Part A: Applied Science and Manufacturing 33 (2002) 971-980.

[12] S. Takeda, Y. Okabe, N. Takeda, Monitoring of delamination growth in CFRP laminates using chirped FBG sensors, Journal of Intelligent Material Systems and Structures 19 (2008) 437-444.

[13] C.S. Lee, W. Hwang, H.C. Park, K.S. Han, Failure of carbon/epoxy composite tubes under combined axial and torsional loading 1. Experimental results and prediction of biaxial strength by the use of neural networks, Composites Science and Technology 59 (1999) 1779-1788.

[14] L. Sorensen, J. Botsis, T. Gmur, J. Cugnoni, Delamination detection and characterisation of bridging tractions using long FBG optica sensors, Composites Part A: Applied Science and Manufacturing 38 (2007) 2087-2096.

[15] G.C. Kahandawa, J.A. Epaarachchi, H. Wang, J. Canning, Effects of the self distortions of embedded FBG sensors on spectral response due to torsional and combined loads, APWSHM3, Tokyo, Japan, 2010.

[16] E. Lewis, C. Sheridan, M. O'Farrell, D. King C. Flanagan, W.B. Lyons, et al., Principal component analysis and artificial neural network based approach to analysing optical fibre sensors signals, Sensors and Actuators A: Physical 136 (2007) 28-38.

[17] P.A.M. Lopes, H.M. Gomes, A.M. Awruch, Reliability analysis of laminated composite structures using finite elements and neural networks, Composite Structures 92 1603-1613.

[18] C.L.N. Veiga, L.S. Encinas, A.C. Zimmermann, Neural networks improving robustness on fiber Bragg gratings interrogation systems under optical power variations, in: D.S. David (Ed.), SPIE 2008, pp. 700462 
[19] A.C. Zimmermann, C. Veiga, L.S. Encinas, Unambiguous signal processing and measuring range extension for fiber bragg gratings sensors using artificial neural networks - a temperature case, Sensors Journal, IEEE 8 (2008) 1229-1235.

[20] L.C.S. Nunes, B.S. Olivieri, C.C. Kato, L.C.G. Valente, A.M.B. Braga, FBG sensor multiplexing system based on the TDM and fixed filters approach, Sensors and Actuators A: Physical 138 (2007) 341-349.

[21] L.C.S. Nunes, L.C.G. Valente, A.M.B. Braga, Analysis of a demodulation system for fiber bragg grating sensors using two fixed filters, Optics and Lasers in Engineering 42 (2004) 529-542.

[22] G.C. Kahandawa, J.A. Epaarachchi, H. Wang, Identification of distrotions to FBG spectrum using FBG fixed filters, ICCM18, jeju Island, Korea, 2011

[23] A. Hosni Elhewy, E. Mesbahi, Y. Pu, Reliability analysis of structures using neural network method, Probabilistic Engineering Mechanics 21 (2006) 44-53.

[24] A.K. Garg, D. Roy Mahapatra, S. Suresh, S. Gopalakrishnan, S.N. Omkar, Estimation of composite damage model parameters using spectral finite element and neural network, Composites Science and Technology 64 (2004) 2477-2493.

[25] J. Zhang, H. Zhao, X.-w. Rong, Application of BP neural network in FBG sensing system performance improvement, electronic packaging technology and high density packaging, 2008, ICEPTHDP 2008, International Conference, 2008, pp. 1-4.

[26] A.S. Paterno, J.C.C. Silva, M.S. Milczewski, L.V.R. Arruda, H.J. Kalinowski, Radial-basis function network for the approximation of FBG sensor spectra with distorted peaks, Measurement Science and Technology 17 (2006) 1039.

[27] A.M. Khathate, G.M. Kamath, M.S. Rao, Sensor failure detection for robustness of shm using a combined artificial neural network and finite element analysis approach, ISSS-MEMS, India, 2007.

[28] T.A. Hamdalla, M.Y. El-Bakry, M.A. Moussa, Fiber Bragg grating as temperature sensor using neural network modeling, International Journal of Scientific and Engineering Research 3 (2012) 351-355. 